\title{
New Mathematical Models for the Mouse Atrial Fast Sodium Channel
}

\author{
Shanzhuo Zhang ${ }^{1}$, Wei Wang ${ }^{1,2}$, Kuanquan Wang ${ }^{1}$, Henggui Zhang ${ }^{1,2,3,4}$ \\ ${ }^{1}$ School of Computer Science and Technology, Harbin Institute of Technology, Harbin, China \\ ${ }^{2}$ Peng Cheng, Laboratory, Shenzhen, China \\ ${ }^{3}$ School of Physics and Astronomy, The University of Manchester, Manchester, UK \\ ${ }^{4}$ Space Institute of Southern China, Shenzhen, China
}

\begin{abstract}
The fast sodium channel (FSC) is one of the most important channels in the cardiomyocytes. It leads the activation of the cardiac action potentials and its dysfunction may leads to many severe pathologies. However, the currently widely used FSC model is not developed for mouse, and relatively outdated compared with the emerging experimental data on mouse atria, making the model less reliable in investigating the mechanisms underlying atrial arrhythmias. In this work, we intend to develop a new model for the mouse atrial FSC which can reproduce the newly published experimental data. The kinetics of and the current generated by our new model were thoroughly validated. We investigated the response of the new model to infra-or supra-threshold stimuli and found that it needs a smaller stimulus to be activated and has a higher driving ability compared with the old model. The current amplitude of the new model also shows a smoother stimulus-dependent curve than the old model. This model will be a more suitable tool in the research of atrial arrhythmias.
\end{abstract}

\section{Introduction}

The fast sodium channel (FSC) plays an important role in the initiation of cardiac action potentials (APs), it activates first in an AP and leads the whole process of cell activation and various downstream cell behaviours. Dysfunction of FSC underlies many severe pathologies, such as congenital and drug-acquired long QT syndromes (LQTS), Brugada syndrome (BrS), and atrial fibrillation [1].

However, models of FSC adopted in recent mouse single myocyte models are relatively outdated. One of currently widely used cardiac FSC models was first proposed by Beeler and Reuter [2]. They introduced the second inactivation gate $\mathrm{j}$ following the suggestion by Haas et al. [3]. Then this model was adopted and modified by Luo \& Rudy, and incorporated in their mammalian ventricular cell model [4], [5]. This model was further slightly modified by Shannon et al. in their rabbit cardiac cell model [6] and used by our mouse atrial cell model [7]. Although endeavors have been taken in the evolution of the FSC model, there has been no major modification on this Hudgkin and Huxley $(\mathrm{HH})$ formulated FSC model since 1990s, and the model was usually fitted to experiment data of other species.

On the other hand, recent experiments have shown that there are obvious discrepancies in FSC kinetics between ventricles and atria. Shekhar et al. [8], [9] found that the sodium current in mouse right atria shows a larger whole cell current, and the activation and inactivation curve are all shifted to lower membrane potentials then that in mouse ventricles.

In this modelling study, we aim at developing a new FSC model with recently published experimental data on mouse atrial FSCs and investigating how this atrialventricular heterogeneity will affect the AP.

\section{Model development}

Our model of FSC adopted classic $\mathrm{HH}$ formalisations, which has three gates, $m$ for activation and $h, j$ for inactivation.

We first fitted steady state activation and inactivation curves for the three gates as shown in Figure $1 \mathrm{~A} \& \mathrm{~B}$. Because of different experiment conditions, data points need to be adjusted for two reasons before usage: temperature adjustment from room temperature to body temperature, and time adjustment for the shifted activation and inactivation curve along with time after the patch rupture. Therefore, the experiment data from Shekhar et al. and Cerrone et al. [9], [10] were first adjust with +12 $\mathrm{mV}$ from room temperature to body temperature (the $\mathrm{V}_{1 / 2}$ of the activation gate was moved from $-57 \mathrm{mV}$ at $16{ }^{\circ} \mathrm{C}$ to $-49 \mathrm{mV}$ at $26{ }^{\circ} \mathrm{C}$ reported by Murray et al. [11], and linearity of the change was assumed). Then the data from 
Shekhar et al. was further moved by $+1.6 \mathrm{mV}$ since the average patch life in their experiments was $15 \mathrm{~min}$ and it was reported that in guinea pig atria the activation curve of FSC move about $+3.2 \mathrm{mV}$ per $1 / 2$ hour [12]. The two inactivation gates share the same steady state inactivation curve. The data was also adjusted for the same reason as the activation gate. Both experiment data sets were shifted by $+10.5 \mathrm{mV}$ for temperature and the data from Shekhar et al. was moved by $+2 \mathrm{mV}$ for time after patch rupture. Our model well fitted in the experimental data.

The time constants of the three gates from our model and experiments are shown in Figure $1 C \& D$. Since there was no experiments on mouse atria, we fitted our model to the data from rat ventricles [13]. The data of time
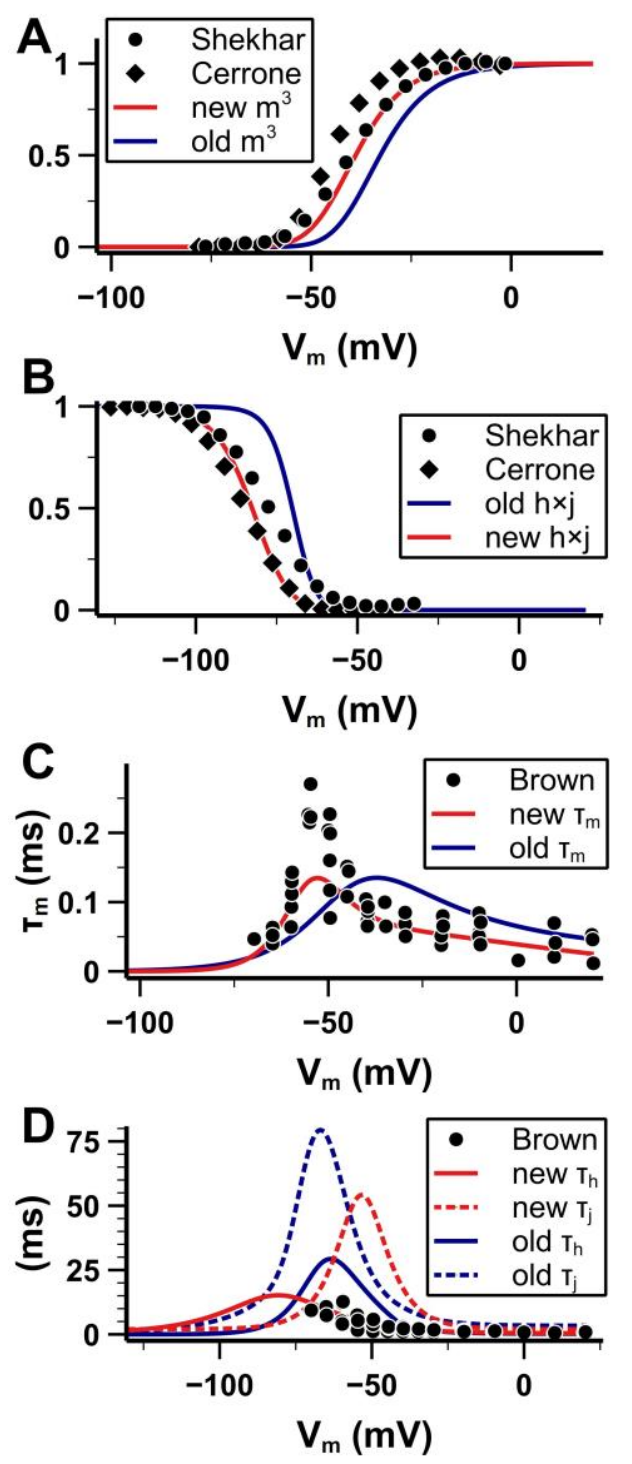

Figure 1. Validation of channel kinetics. (A) Steady state activation. (B) Steady state inactivation. (C) activation time constant. (D) Inactivation time constant. constant for $\mathrm{m}$ and $\mathrm{h}$ was adjusted by a Q10 factor of 0.5 and 0.4 , respectively. The time constants of $m$ and $h$ were in good agreement with the experiment data. The time constant of $\mathrm{j}$ was manually modified due to lack of experimental data.

It has been found in experiments that the conductance of FSC in atria is bigger than that in ventricles. The maximum conductance was $1.14 \pm 0.07 \mathrm{nS} / \mathrm{pF}$ in right atria and $0.92 \pm 0.04 \mathrm{nS} / \mathrm{pF}$ in ventricles [9]. Also considering the maximum upstroke velocity should be around $200 \mathrm{~V} / \mathrm{s}$ and the overshoot about $+20 \mathrm{mV}$, we set the conductance of the FSC to $1.2 \mathrm{nS} / \mathrm{pF}$ at room temperature. $\mathrm{A} \mathrm{Q}_{10}$ factor of $2.36 \pm 0.29$ was used on the conductance [14].

The model equations are listed below:

$$
\begin{gathered}
Q 10_{\text {tau_h_Na }}=1.0 / 2.5 \\
Q_{\text {pow }}=1.5
\end{gathered}
$$
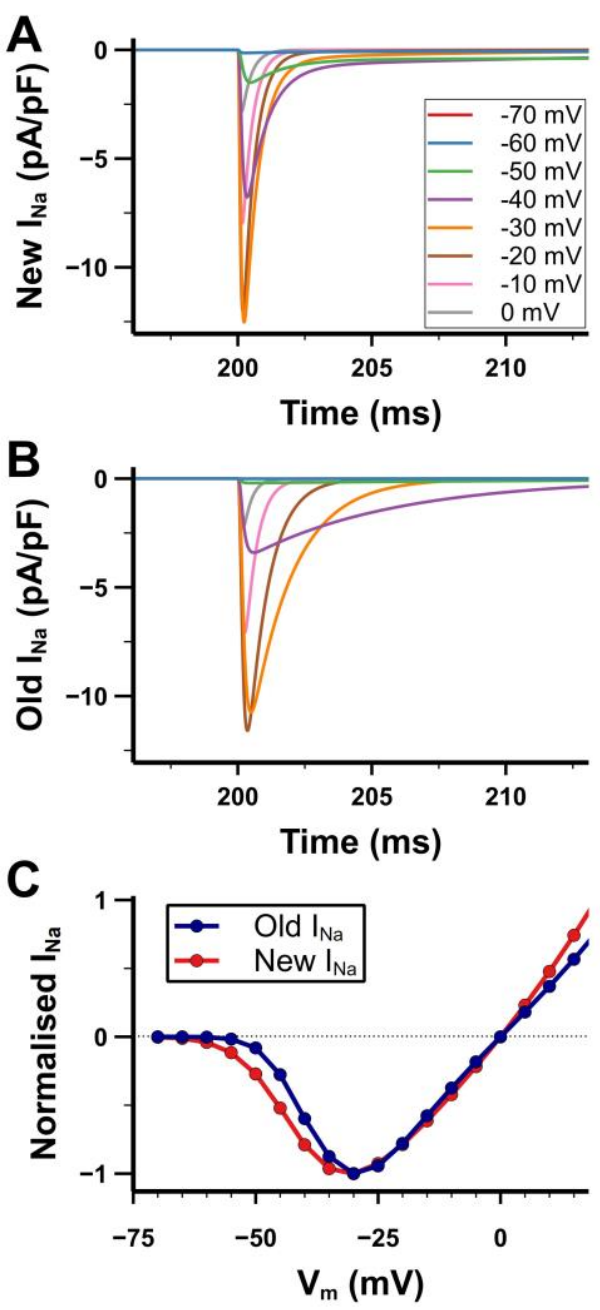

Figure 2. Validation of channel currents under voltageclamp protocols. (A \& B) Currents simulated from holding potential of $-80 \mathrm{mV}$ to indicated test potentials. (C) I-V relationship of the two models of FSC. 


$$
\begin{gathered}
m_{\text {inf }}=\frac{1}{\left(1+e^{-(V+49) / 7.5}\right)} \\
h_{\text {inf }}=\frac{1}{\left(1+e^{(V+76.6) / 6.8}\right)} \\
\operatorname{tau}_{m}=1 /\left(5 \times e^{(V+25) / 27}+13 /\left(1+e^{(-V-45) / 7}\right)\right. \\
\left.+8.6 \times e^{-(V+60.4) / 6}\right) \\
\operatorname{tau}_{h}=0.35+1.4 /\left(1.2 \times e^{-(V+130.2) / 15.3}\right. \\
+0.7 \times e^{(V+39.5) / 15.27} /\left(Q 10_{\text {tau }}{ }^{(N a}\right) \\
\operatorname{tau}_{j}=1+1.3 /\left(2.4 \times e^{-(V+72.2) / 3.3}\right. \\
\left.+0.4 \times e^{(V+34.5) / 4.3}\right) \\
I_{N a}=G_{N a} \times m^{3} \times h \times j \times\left(V-E_{N a}\right)
\end{gathered}
$$

\section{Result}

Simulated current traces for both the old and new FSCs are shown in Figure 2A \& B. The channel was held at $-80 \mathrm{mV}$, then a series of test potentials ranging from -70 $\mathrm{mV}$ to $0 \mathrm{mV}$ with a $10-\mathrm{mV}$ step was applied to activate the
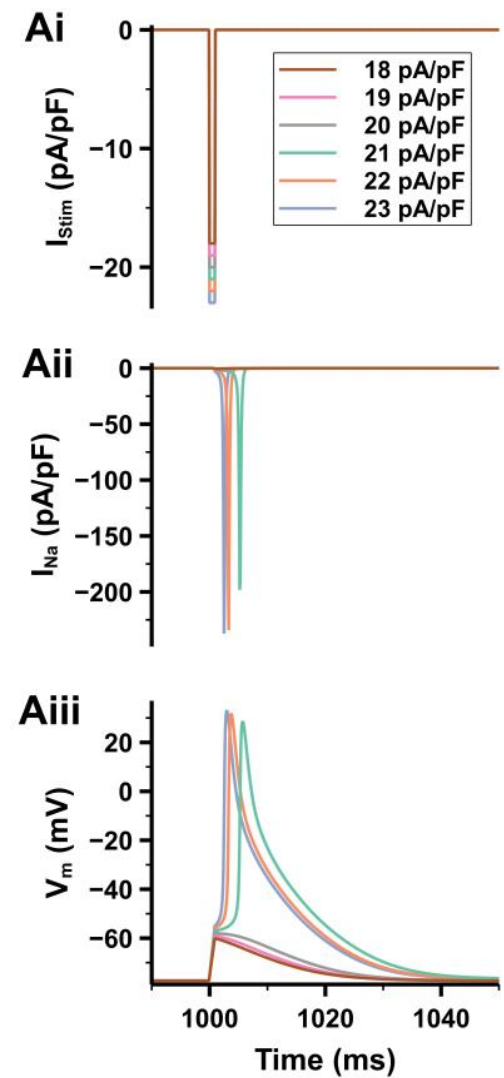

channel. The new model showed almost the same amplitude as the old model, but a shorter total open time. We can see from the Figure 2A that our model was activated at $-50 \mathrm{mV}$ (green line), while the old model was activated at $-40 \mathrm{mV}$. It can also be seen in the I-V relationship shown in Figure 2C that the newly developed model activated at lower membrane potential.

We incorporated the new FSC model into a newly developed mouse atrial cell model to investigate its influence on the activation of APs (Figure 3). Generally, the new FSC model needed a smaller stimulus to initiate an AP. The activation threshold of the new model was 15 $\mathrm{pA} / \mathrm{pF} \times 1 \mathrm{~ms}$, while that of the old model was $21 \mathrm{pA} / \mathrm{pF}$ $\times 1 \mathrm{~ms}$. Another interesting thing was that the current of the old model changed steeply around the threshold. The amplitude of current was about $200 \mathrm{pA} / \mathrm{pF}$ with the suprathreshold stimulus and was nearly $0 \mathrm{pA} / \mathrm{pF}$ with just 1 $\mathrm{pA} / \mathrm{pF}$ below the threshold. On the contrary, the new model had a smoother changing (Figure 3Bii). This smooth changing also reflected on the AP morphology (Figure 3Biii). The APs initiated by the new model showed various upstroke velocity and overshoot.

The maximum sodium current and AP characteristics of the old and new FSC models are measured and shown in Figure 4. The new model was smoother varying from
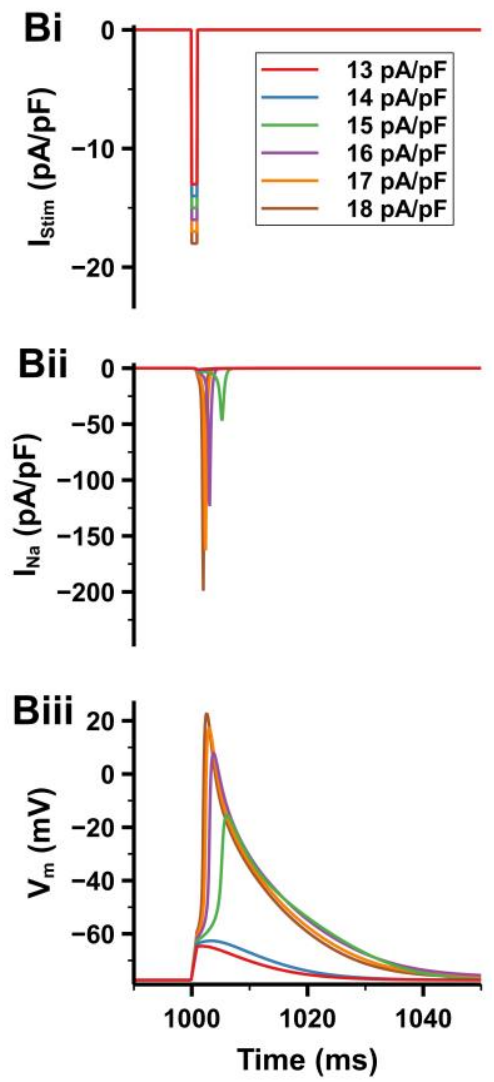

Figure 3. Altered excitability with the new model of FSC. (A) Simulated results from the old model. (B) Simulated results from the new model. (i) Stimulus current in $\mathrm{pA} / \mathrm{pF}$. The duration of all stimuli was 1 ms. (ii) The fast sodium current. (iii) Action potentials with two different models of FSC. 
the threshold and maximum amplitude (Figure 4A). And it led to a higher overshoot in the whole range of stimulus (Figure 4B), indicating a stronger driving ability. $\mathrm{APD}_{30}$ and $\mathrm{APD}_{90}$ were both in a reasonable range.

\section{Discussion and conclusion}

In this work, we developed a new FSC model specifically for the mouse atrial myocyte based on newly published experimental data. Following other FSC models, our model adopted the classic $\mathrm{HH}$ formalisation, but better mimic the kinetics of mouse atrial sodium current. Compared with the old FSC model, our model replicated the property that the mouse atrial FSC activates at a lower membrane potential, which leads to a higher excitability
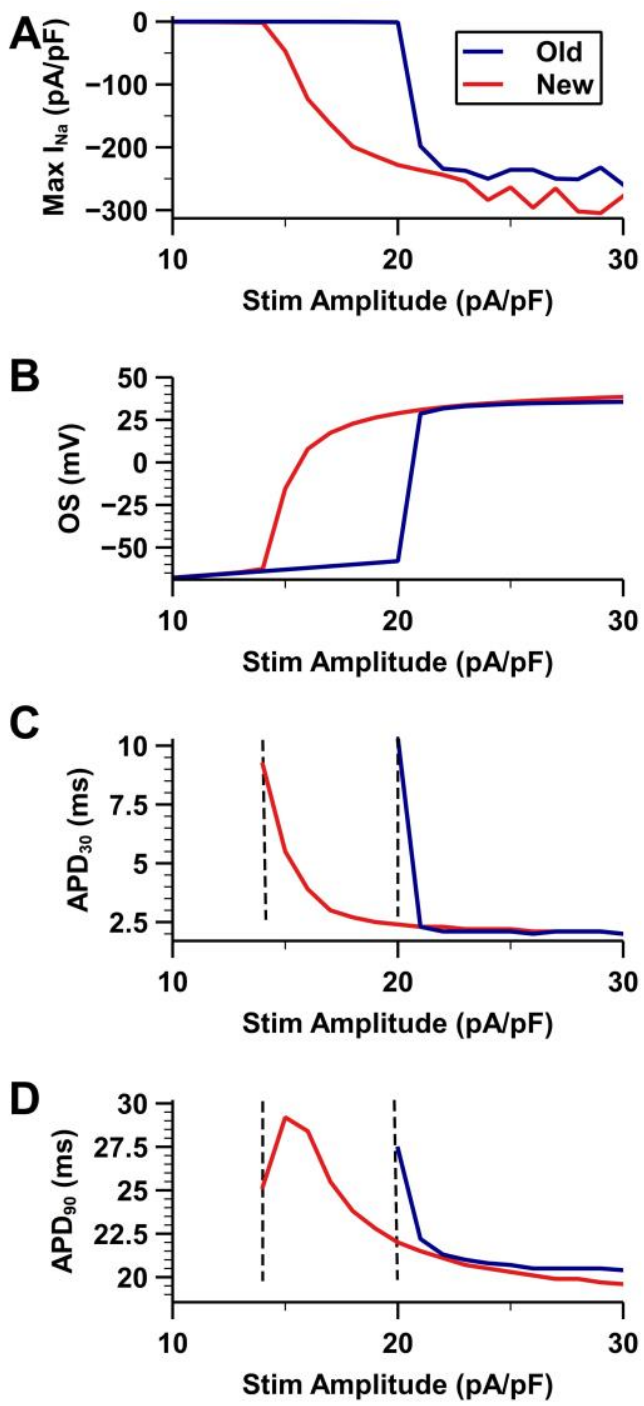

Figure 4. The sodium current and AP characteristics under various stimulus amplitudes. (A) Maximum sodium current. (B) Overshoot. (C \& D) $\mathrm{APD}_{30}$ and $\mathrm{APD}_{90}$. The curve was cut beyond the dash lines since the cell failed to be activated by small stimulus. (smaller stimulus needed) and smoother activation process.

\section{References}

[1] H. Abriel, "Roles and regulation of the cardiac sodium channel Nav1.5: Recent insights from experimental studies," Cardiovasc Res, vol. 76, no. 3, pp. 381-389, Dec. 2007.

[2] G. W. Beeler and H. Reuter, "Reconstruction of the action potential of ventricular myocardial fibres," J Physiol, vol. 268, no. 1, pp. 177-210, 1977.

[3] H. G. Haas, R. Kern, H. M. Einwächter, and M. Tarr, "Kinetics of $\mathrm{Na}$ inactivation in frog atria," Pflugers Arch, vol. 323, no. 2, pp. 141-157, Jun. 1971.

[4] C. Luo and Y. Rudy, "A model of the ventricular cardiac action potential. Depolarization, repolarization, and their interaction," Circ Res, vol. 68, no. 6, pp. 1501-1526, 1991.

[5] C. Luo and Y. Rudy, "A dynamic model of the cardiac ventricular action potential. I. Simulations of ionic currents and concentration changes," Circ Res, vol. 74, no. 6, pp. 1071-1096, 1994.

[6] T. R. Shannon, F. Wang, J. Puglisi, C. Weber, and D. M. Bers, "A mathematical treatment of integrated Ca dynamics within the ventricular myocyte," Biophys $J$, vol. 87, no. 5, pp. 3351-3371, Nov. 2004.

[7] W. Shen, "Development of a biophysically detailed mathematical model of a mouse atrial cell for the study of cellular proarrhythmic mechanisms," University of Manchester, 2016.

[8] A. Shekhar et al., "Transcription factor ETV1 is essential for rapid conduction in the heart," J Clin Invest, vol. 126, no. 12 , pp. 4444-4459, Dec. 2016.

[9] A. Shekhar et al., "ETV1 activates a rapid conduction transcriptional program in rodent and human cardiomyocytes," Sci Rep, vol. 8, no. 1, p. 9944, Jul. 2018.

[10] M. Cerrone et al., "Sodium current deficit and arrhythmogenesis in a murine model of plakophilin-2 haploinsufficiency," Cardiovasc Res, vol. 95, no. 4, pp. 460-468, Sep. 2012.

[11] K. T. Murray, T. Anno, P. B. Bennett, and L. M. Hondeghem, "Voltage clamp of the cardiac sodium current at 37 degrees $\mathrm{C}$ in physiologic solutions.," Biophys $J$, vol. 57, no. 3, pp. 607-613, Mar. 1990.

[12] G.-R. Li, C.-P. Lau, and A. Shrier, "Heterogeneity of sodium current in atrial vs epicardial ventricular myocytes of adult guinea pig hearts," J Mol Cell Cardiol, vol. 34, no. 9, pp. 1185-1194, Sep. 2002.

[13] A. M. Brown, K. S. Lee, and T. Powell, "Sodium current in single rat heart muscle cells.," J Physiol, vol. 318, pp. 479500, Sep. 1981.

[14] M. Kohlhardt, "Different temperature sensitivity of cardiac $\mathrm{Na}+$ channels in cell-attached and cell-free conditions," Am J Physiol-Cell Ph, vol. 259, no. 4, pp. C599-C604, Oct. 1990.

Address for correspondence:

Shanzhuo Zhang.

Room 306, Building of Integrated Labs, Harbin Institute of Technology, Harbin, China, 150001.

shanzhuo.zhang@gmail.com 